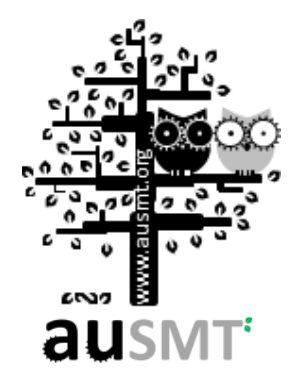

\title{
Multi-sensor Approach for Multivalent Applications in Nanometrology
}

\section{Eberhard Manske*and Gerd Jäger}

IImenau University of Technology, Germany

(Received 12 March; Accepted 26 April 2012; Published on line 1 June 2012)

*Corresponding author: eberhard.manske@tu-ilmenau.de

DOI: $10.5875 / a u s m t . v 2 i 2.149$

\begin{abstract}
The field of nanometrology is subject to increasing complexity as dimensional structures become more intricate with smaller structural widths and increasingly larger surface areas, and with the inclusion of thousands of inspection features. The relevant metrology tools thus need to provide the ability to measure in three dimensions with atomic resolution over large areas, and at a speed/throughput suitable for industrial applications. Ilmenau developed Nanopositioning and Nanomeasuring Technologies with an measuring range of tens of millimeter, subnanometer resolution and nanometer accuracy, allowing for combination with a multi-sensor approach for multivalent measurement and positioning applications in nanometrology.
\end{abstract}

Keywords: Nanopositioning; Nanomeasuring; Nanometrology

\section{Introduction}

The field of nanometrology is subject to increasing complexity as dimensional structures becoming more intricate with smaller structural widths and increasingly larger surface areas, and include thousands of inspection features. The relevant metrology tools thus need to provide the ability to measure in three dimensions with atomic resolution over large areas with speed/throughput suitable for industrial applications. Ilmenau developed Nanopositioning and Nanomeasuring Technologies with a measuring range of tens of millimeter, subnanometer resolution and nanometer accuracy, allowing for combination with a multi-sensor approach for multivalent measurement and positioning applications in nanometrology.

\section{Nanopotitioning and Nanomeasuring Machine}

Working with SIOS Meßtechnik GmbH, the IImenau University of Technology developed a nanopositioning and nanomeasuring machine NMM-1 with a measuring volume of $25 \times 25 \times 5 \mathrm{~mm}^{3}$ and a resolution of $0.1 \mathrm{~nm}$ [1]. Achieving nanometer precision requires a set-up that minimizes errors. Therefore, the basic concept consists of a special arrangement which allows Abbe error free measurements in all measuring axes over the whole measuring range (Figure 1). That means that the distance between the measuring axis and normal axis, the so-called Abbe offset $I_{\text {off, }}$, should be zero in every axis.

$$
\mathrm{I}_{\text {offi }} \rightarrow 0 \forall \mathrm{i} \in<\mathrm{x}, \mathrm{y}, \mathrm{z}>
$$




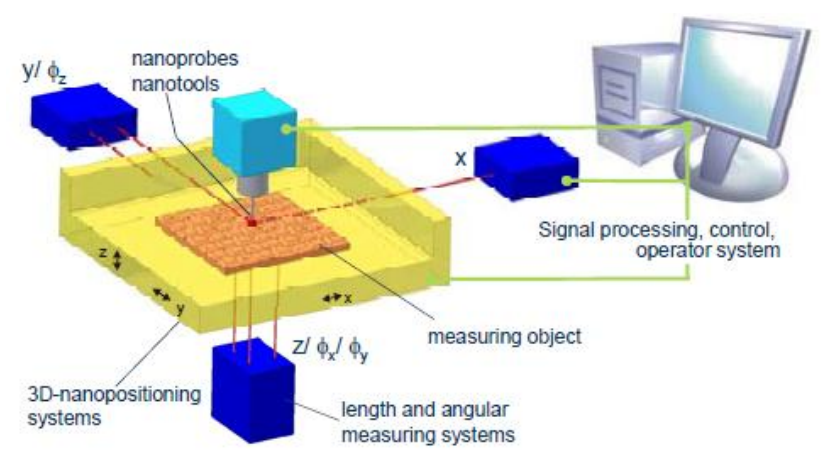

Figure 1. Basic concept and main components of the NPM-machine.

Expanding the Abbe comparator principle not only minimizes the length offset between the measuring axis and the normal axis, but also carries out an active measurement and correction of angular deviations $\alpha_{i}$ around all moving axes.

$$
\alpha_{\mathrm{i}} \rightarrow 0 \forall \mathrm{i} \in<\mathrm{x}, \mathrm{y}, \mathrm{z}>
$$

The Abbe error can be minimized as follows:

$$
\Delta \mathrm{l}_{\mathrm{i}}=\mathrm{I}_{\mathrm{off}_{\mathrm{f}}} \cdot \sin \alpha_{\mathrm{i}} \rightarrow 0 \forall \mathrm{i} \in<\mathrm{x}, \mathrm{y}, \mathrm{z}>
$$

Prof. Dr.-Ing. habil. Eberhard Manske has held a foundation professorship in "Precision Measurement Technology" at the Ilmenau University of Technology since 2008. He has served as the spokesman of the collaborative research centre "SFB 622 - Nanopositioning and Nanomeasuring Machines" since June 2008, succeeding the initiator and long-standing head, Prof. Gerd Jäger.

Since 2010 he has served as interim head of the Mechanical Engineering Measurement and Production Measurement department.

After the completion of his studies in electrical engineering in 1982, Prof Manske worked at the Institute of Process Measurement and Sensor Technology at the IImenau University of Technology. In 1986, he obtained his doctoral degree, and in $\mathbf{2 0 0 6}$ his postdoctoral lecturing qualification.

His research focuses mainly on nanopositioning and nanomeasuring technology, fibre-coupled laser interferometry, laser stabilization, optical and tactile precision sensors and scanning probe techniques.

Prof. Gerd Jäger is a University-Professor at the Technical University IImenau in Germany and also a guest professor at Tianjin University and the Xian University of Technology in China. He received his Ph.D. from the Technical University Ilmenau in 1970. From 1974 to 1975 he was a guest researcher at the National Technical University of Ukraine. Since 1981 he has served as Professor and director of the Institute of Process Measurement and Sensor Technology at TU IImenau.

In 2002 Prof. Jäger initiated and established the collaborative research centre "SFB 622 - Nanopositioning and Nanomeasuring Machines" at IImenau University of Technology. He was the spokesman of this CRC until 2008.

In 1998 he was awarded an honorary doctorate (h.c.) by the National Technical University of Ukraine in Kiev. In 2002 he was awarded an honorary doctorate by the National University Lvivska Politechnika in Lviv. He is a fellow of the Academy Erfurt in Germany.

His research fields and lectures include "Process Measurement and Sensor Technology", "Laser-based Nanomeasuring and -positioning Technology", "Force Measurement and Weighing Technique" and "Temperature Measurement". In 1991 he founded the SIOS measurement technology company.

Prof. Jäger has published about 60 papers in engineering journals, has written chapters for four engineering coursebooks, has registered about 40 patents and has presented at over 160 national and international conferences. During the last decade Prof. Jäger has held some guest lecturing positions in Germany, Ukraine, China, Taiwan and the USA.

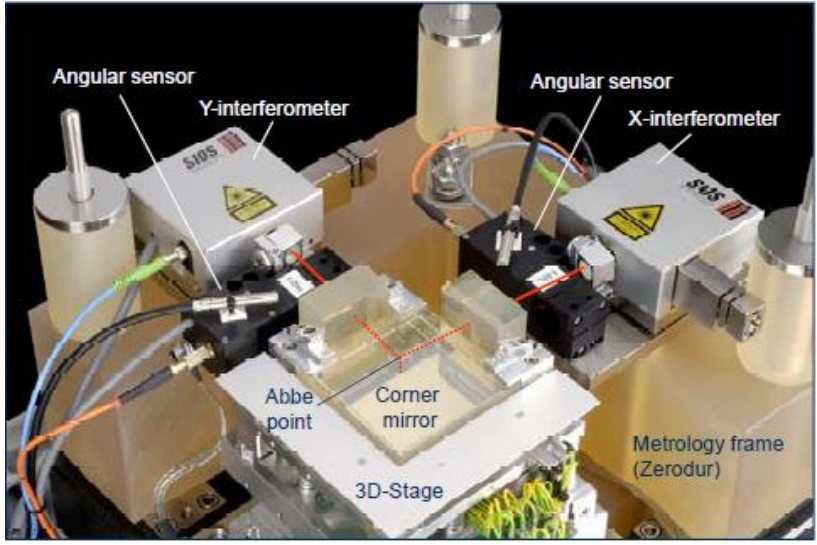

Figure 2. Basic components of the NMM-1.

Such a set-up can be realized particularly by an arrangement in which the nanoprobe works as a null indicator and whose scanning point lies in the intersection of the three coordinate measuring axes of three laser interferometers (Figure 1).

The laser beams are reflected on a so-called mirror corner which represents the orthogonal co-ordinate system and which carries the object to be measured. The mirror corner, together with the object to be measured, is moved in a closed-loop control by a 3D-precision stage (Figure 2).

Additional angular sensors measure the stage's angular deviations, which are compensated by four $z$-drives. The measuring systems are mounted on a mechanical and thermal stable metrology frame (Zerodur). This arrangement forms a universal and advanced platform for high precision measurement tasks for several nanoprobing systems.

\section{Multisensor Approach}

The broad variety of measurement tasks requires the use of several probes with different measurement properties. Frequently changing complete probe systems is inconvenient but certain specimens with several features can only be measured with special probes. Therefore, a multisensor approach was developed based on an optical focus sensor. Here, a modular assembly is used to integrate five sensors. Probes can be changed easily and quickly with a microscope revolver.

The focus sensor itself is based on a so-called hologram-laser-unit (Figure 3 ) and has a spot diameter of $0.6 \mu \mathrm{m}$, a numerical aperture of 0.55 , and a vertical resolution of $<1 \mathrm{~nm}$. This sensor allows scanning speeds of up to $6 \mathrm{~mm} / \mathrm{s}$ on moderate objects at a reproducibility of $<2 \mathrm{~nm}$, which cannot be achieved using tactile sensors. 

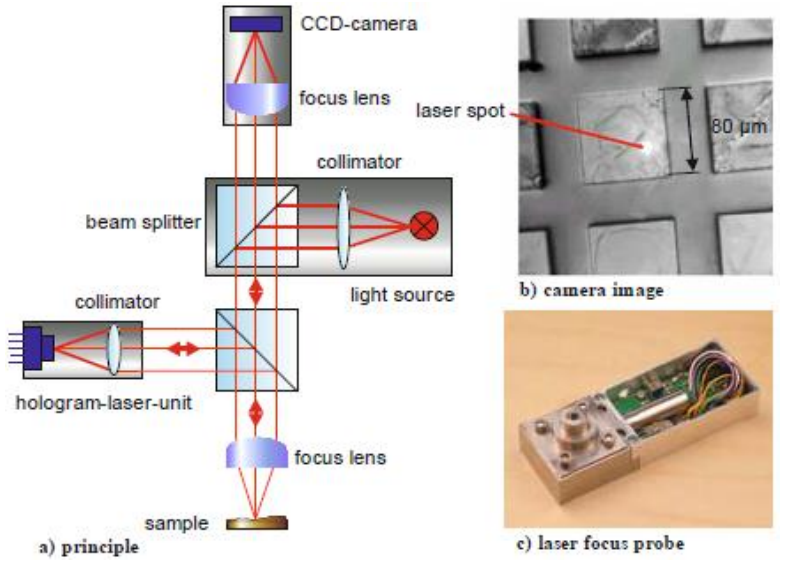

Figure 3. Laser focus probe.
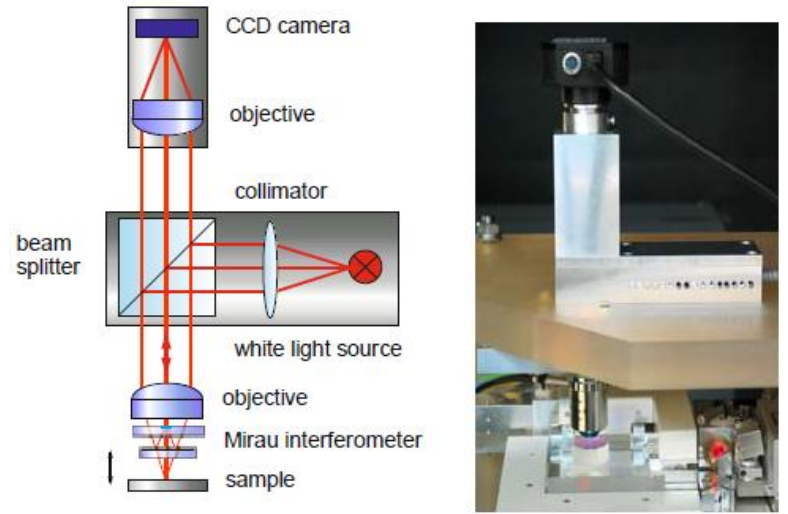

Figure 4. White-light interference microscope: Principle and integration in the NPM machine.

The laser focus probe is a single point sensor. The measuring data are recorded sequentially and point by point, thus allowing for highly variable measurements. However, the scanning of particularly large surfaces with a high point density can be very time-consuming. Therefore, the focus sensor was modified as an interference microscope (Figure 4). The focus lens is replaced by a Mirau interferometer objective, and the viewing microscope is now directly used for recording the measuring data. Using a 20-fold objective, about 750,000 measuring points can be captured and processed simultaneously in one field of view $(0.8 \mathrm{~mm} x$ $0.6 \mathrm{~mm}$ ) with a CCD camera, with a pixel distance of 0.8 $\mu \mathrm{m}$. On average, one z-scan takes about $20 \mathrm{~s}$, as opposed to at least 30 minutes for scanning the same amount of data by means of a single point sensor. In comparison with conventional white-light microscopes, the z-scan is performed with the interferometric precision of the NPM machine. In principle, step heights of up to $5 \mathrm{~mm}$ can be measured (limited at the moment to $3 \mathrm{~mm}$ by the working distance of the Mirau objective ) - compared to a measuring range of $100 \mu \mathrm{m}$ in the case of conventional interference microscopes. Furthermore, it is possible to measure larger surfaces by sequentially stitching together smaller surface profiles. These individual "height maps" can be joined together with nanometer precision via the NPM machine. Thus, for example, a measuring surface of $4 \mathrm{~mm} \times 4 \mathrm{~mm}$ consisting of 64 single maps including 21 million data points can be measured in less than 25 minutes.

However, while this sensor offer the advantage of non-contact measurement, it also presents the typical disadvantages of optical sensors such as diffraction phenomena appearing on steep edges and phase jumps on different materials. The lateral resolution is also limited by the diffraction-dependent spot diameter.

Nevertheless, we can use the high resolution of the laser focus sensor to assemble tactile probing systems. The focus sensor was first combined with the stylus of a tactile profilometer (Figure 5 , center), with the laser beam focused on the back of the stylus. The measuring surface is scanned with a conventional diamond-tipped stylus (scanning tip diameter of $2 \mu \mathrm{m}$, slope angle: $90^{\circ}$ ). The tactile sensor works in a purely passive mode. The combined action of the integrated spring and the weight of the stylus results in a constant measurement force of $<0.1 \mathrm{mN}$. A big advantage of this arrangement in comparison with conventional tactile profilometer systems is that the sensor works as a null indicator, thus minimizing measuring force fluctuations and measurement deviations resulting from the bearing clearance of the pivot. Depending on the probe surface type, a scanning speed of $10-100 \mu \mathrm{m}$ can be achieved in the scanning mode, with measurement uncertainty $<1$ $\mathrm{nm}$. However, the lateral resolution of the sensor is again limited by the diameter of the stylus tip.

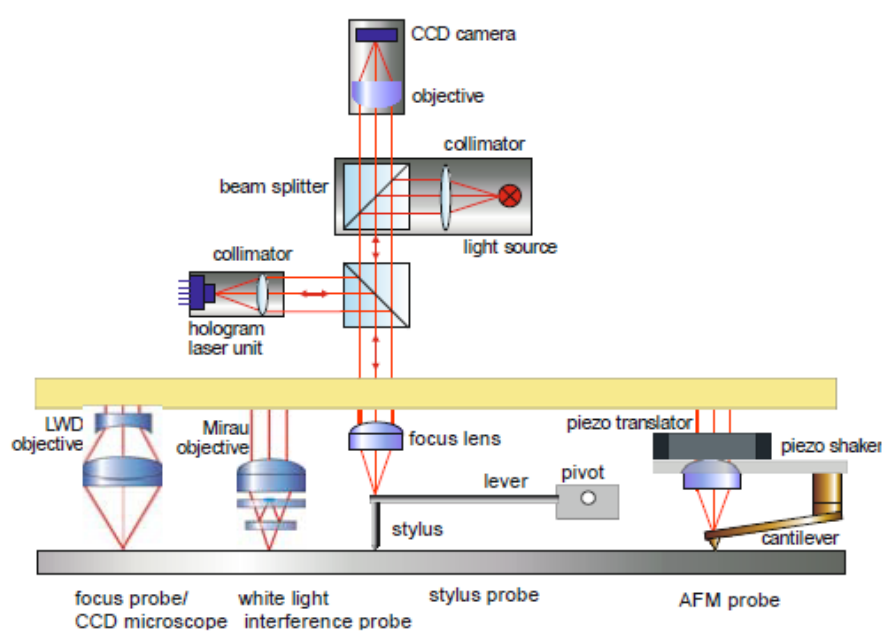

Figure 5. Several probing systems based on laser focus probe. 


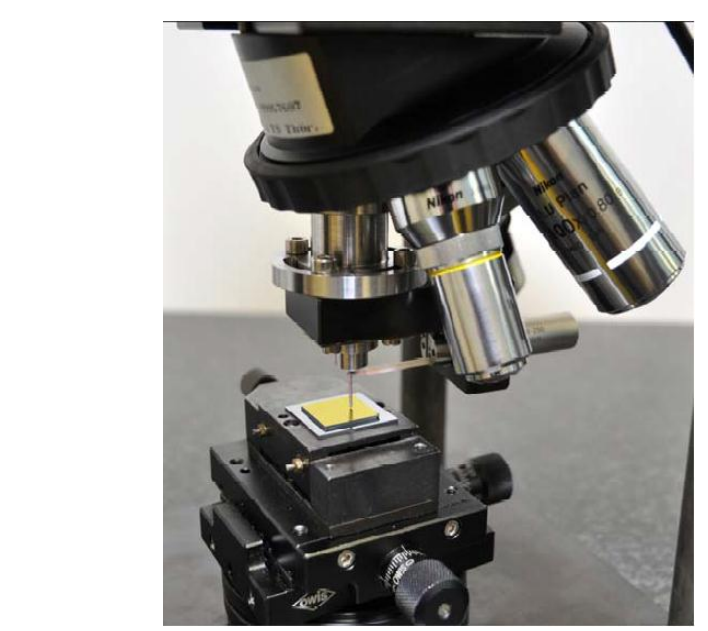

Figure 6. Microscope revolver approach.

To be able to scan real micro- and nanostructures, the scanning radius must be reduced considerably. This can be achieved by applying the scanning force probe technique with tip radii of $<10 \mathrm{~nm}$. For this, an AFM-cantilever was again combined with the focus sensor (Figure 5, right). Thus, this system operates as an atomic force sensor. The focus sensor detects the deflection of the cantilever due to atomic forces which become effective when the stylus approaches the object to be measured. During the SFM measurement, the force acting between the tip and the sample is weak and, even in the contact mode, it reaches only a few nanonewtons. This prevents scratching of the measured surface during the SFM scanning process, as opposed to stylus profilometers which may scratch soft material surfaces. An additional piezoelectric element serves as shaker for the dynamic scanning mode.

If this atomic force sensor is used in the nanomeasuring machine exclusively as a null indicator, the achievable scanning speed will be limited to several $\mu \mathrm{m} / \mathrm{s}$ by the high mass of the NPM machine's $x-y-z$ table. Therefore, a further piezotranslator with an integrated length sensor is used to provide the quick z-movement of the cantilever and of the focus lens, which is rigidly connected with the piezotranslator. Combining the quick z-movement in the AFM-head and the slower movement of the heavy measuring table achieves a considerable improvement of the measuring dynamics. Depending on the surface structure of the object to be measured, scanning speeds of up to $100 \mu \mathrm{m} / \mathrm{s}$ were realized.

Due to the fact that the measuring tasks to be solved are highly varied and present quite different requirements regarding the size of the measuring field, the lateral resolution, the point density, and the application of an optical or tactile method, a combination of the various sensor technologies is desirable.
The arrangement shown in Figure 5 can be used as laser focus sensor, measuring microscope, white-light interference microscope, tactile stylus sensor or as an AFM probe. All arrangements are based on the laser focus probe combined with a CCD-camera microscope. The microscope can be used together with the focus probe through an additional revolver system to accommodate the various scanning systems, thus making it possible to quickly and easily switch mechanically to the different sensors.

Such revolver systems are also available with a motor drive, but these systems are not designed for high reproducibility between single revolver positions. Combining the measuring data gathered by different sensors on a given object requires determining the exact relation between the single measurements. This shall be realized by means of fiducial marks which are attached either to the measuring table or to the measuring object itself.

On the basis of the revolver system combined with the fiducial mark technique, various measuring strategies dependant on specific measuring tasks are developed and investigated by different sensor combinations. The next challenge is the development of automatic probe selection as well as computer-aided inspection plan generation. However, problems can arise if the topography or structures are not known from the available CAD data. In some cases an automatic image capturing procedure for the full measuring area (e.g., 25 $\mathrm{mm} \times 25 \mathrm{~mm}$ ) can be carried out in less than 30 minutes. These 2000 single images can be stitched together with nanometer precision via the NPM machine through automated routines to generate an overall "map" of the surface with about 3 GPixels of data and a measurement resolution of $0.8 \mu \mathrm{m}$ [2]. The whole image file can be segmented to highlight interesting object characteristics, producing an overview of all those areas which present certain surface characteristics, and allowing these areas to be considered more closely for an AFM scan.

The NPM machine is currently able to navigate in reality on the basis of this virtual map with nanometer precision. The fields of interest can be approached in a well-directed way and then scanned by the AFM, thus, drastically reducing measurement times and increasing measurement feasibility.

\section{Summary}

The nanopositioning and nanomeasuring machine requires a multi-sensor set-up to measure micro- and nanostructures. Based on a laser focus sensor, different optical, tactile and AFM measuring principles can be realized at nanometer reproducibility and can be 
combined to form a unified multi-sensor system. At the same time, the large number of test features on microand nanostructures requires new measuring strategies to obtain measurements quickly. Automatic probe selection as well as computer-aided inspection plan generation is the next challenge.

\section{Acknowledgement}

The authors wish to thank all their co-workers from the Collaborative Research Centre SFB 622 "Nanopositioning and nanomeasuring machines" and SIOS Meßtechnik GmbH for their contributions, and the German Research Foundation for funding.

\section{References}

[1] E. Manske, T. Hausotte, R. Mastylo, T. Machleidt, K. $H$. Franke, and G. Jäger, "New applications of the nanopositioning and nanomeasuring machine by using advanced tactile and non-tactile probes," Measurement Science and Technology, vol. 18, no. 2, p. 520, 2007. doi: $10.1088 / 0957-0233 / 18 / 2 / S 27$
[2] T. Machleidt, E. Sparrer, N. Dorozhovets, E. Manske, K.-H. Franke, and D. Kapusi, "Navigation in a large measurement volume by using afm technology as a sensor system in the npmm navigation in einem großen messvolumen in der nanopositionier- und mess-maschine (NPMM) mittels AFM-technologie," TM - Technisches Messen, vol. 76, no. 5, pp. 274-277, 2009. doi: $\underline{10.1524 / \text { teme. } 2009.0968}$

[3] International technology roadmap for semiconductors - metrology, 2009, [Online]. Available:

http://www.itrs.net/Links/2009ITRS/2009Chapters 2009Tables/2009 Metrology.pdf 